\title{
Asymptotic stability equals exponential stability, and ISS equals finite energy gain - if you twist your eyes
}

\author{
Lars Grüne * \\ Fachbereich Mathematik, J.W. Goethe-Universität, Postfach 1119 32, D-60054 Frankfurt a.M., \\ Germany, E-Mail: gruene@math.uni-frankfurt.de

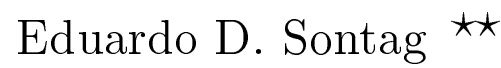 \\ Department of Mathematics, Rutgers University, New Brunswick, NJ 08903, USA, \\ E-Mail: sontag@control.rutgers.edu \\ Fabian R. Wirth \\ Zentrum für Technomathematik, Universität Bremen, D-28344 Bremen, Germany, \\ E-Mail: fabian@math.uni-bremen.de
}

In this paper we show that uniformly global asymptotic stability for a family of ordinary differential equations is equivalent to uniformly global exponential stability under a suitable nonlinear change of variables. The same is shown for input-to-state stability and input-to-state exponential stability, and for input-to-state exponential stability and a nonlinear $H_{\infty}$ estimate.

Key words: asymptotic stability, exponential stability, input-to-state stability, nonlinear $H_{\infty}$

\section{Introduction}

Lyapunov's notion of (global) asymptotic stability of an equilibrium is a key concept in the qualitative theory of differential equations and nonlinear control. In general, a far stronger property is that of exponential stability, which requires decay estimates of the type " $\|x(t)\| \leq$ $c e^{-\lambda t}\|x(0)\| . "$ (See for instance [16] for detailed discussions of the comparative roles of asymptotic and exponential stability in control theory.) In this paper, we show that, for differential equations evolving in finite-dimensional Euclidean spaces $\mathbb{R}^{n}$ (at least in spaces of dimensions $\neq 4,5)$ the two notions are one and the same under coordinate changes.

‡ This paper has been written while the first author was visiting the Dipartimento di Matematica, Universitá di Roma "La Sapienza", Italy, supported by DFG-Grant GR1569/2-1.

${ }^{\star}$ Supported in part by US Air Force Grant F49620-98-1-0242 
Of course, one must define "coordinate change" with care, since under diffeomorphisms the character of the linearization at the equilibrium (which we take to be the origin) is invariant. However, if, in the spirit of both structural stability and the classical HartmanGrobman Theorem (which, cf. [23], gives in essence a local version of our result in the special hyperbolic case), we relax the requirement that the change of variables be smooth at the origin, then all obstructions disappear. Thus, we ask that transformations be infinitely differentiable except possibly at the origin, where they are just continuously differentiable. Their respective inverses are continuous globally, and infinitely differentiable away from the origin.

Closely related to our work is the fact that all asymptotically stable linear systems are equivalent (in the sense just discussed) to $\dot{x}=-x$; see e.g. [1]. The basic idea of the proof in [1] is based upon projections on the level sets of Lyapunov functions, which in the linear case of course be taken to be quadratic (and hence have ellipsoids as level sets). It is natural to use these ideas also in the general nonlinear case, and Wilson's paper [36], often cited in control theory, remarked that level sets of Lyapunov functions are always homotopically equivalent to spheres. Indeed, it is possible to obtain, in great generality, a change of coordinates rendering the system in normal form $\dot{x}=-x$ (and hence exponentially stable), and several partial versions of this fact have appeared in the literature, especially in the context of generalized notions of homogeneity for nonlinear systems; see for instance [6,25,15,27,24].

It is perhaps surprising that, at least for unperturbed systems, the full result seems not to have been observed before, as the proof is a fairly easy application of results from differential topology. (Those results are nontrivial, and are related to the generalized Poincaré conjecture and cobordism theory; in fact, the reason that we only make an assertion for $\neq 4,5$ is closely related to the fact that the original Poincaré conjecture is still open.)

Note, however, that it has been common practice in the papers treating the nonlinear case to use the flow generated by the original system to define an equivalence transformation, thereby reducing the regularity of the transformation to that of the system. Here we use the flow generated by the (normalized) Lyapunov function itself, which yields more regular transformations. In addition, and most importantly, our poof also allows for the treatment of perturbed systems (for which the reduction to $\dot{x}=-x$ makes no sense).

Lyapunov's notion is the appropriate generalization of exponential stability to nonlinear differential equations. For systems with inputs, the notion of input to state stability (ISS) introduced in [29] and developed further in [5,9,13,14,17,18,26,28,32,33] and other references, has been proposed as a nonlinear generalization of the requirement of finite $\mathcal{L}^{2}$ gain or, as often also termed because of the spectral characterizations valid for linear systems, "finite nonlinear $H^{\infty}$ gain" (for which see e.g. [2,11,12,34]). We also show in this paper that under coordinate changes (now in both state and input variables), the two properties (ISS and finite $H^{\infty}$ gain) coincide (again, assuming dimension $\neq 4,5$ ).

We do not wish to speculate about the implications of the material presented here. Obviously, there are no "practical" consequences, since finding a transformation into an exponentially stable system is no easier than establishing stability (via a Lyapunov function). Perhaps these remarks will be of some use in the further theoretical development of ISS and other stability questions. In any case, they serve to further justify the naturality of Lyapunov's 
ideas and of concepts derived from his work.

\section{Setup}

We consider the family of differential equations

$$
\dot{x}(t)=f(x(t), d(t))
$$

where $f: \mathbb{R}^{n} \times D \rightarrow \mathbb{R}^{n}$ is continuous and for $x \neq 0$ locally Lipschitz continuous in $x$, where the local Lipschitz constants can be chosen uniformly in $d \in D \subseteq \mathbb{R}^{m}$. Let $\mathcal{D}$ denote the set of measurable, locally essentially bounded functions from $\mathbb{R}$ to $D$. For any $x_{0} \in \mathbb{R}^{n}$ and any $d(\cdot) \in \mathcal{D}$, there exists at least one maximal solution of (1) for $t \geq 0$, with $x(0)=x_{0}$. By abuse of notation, we denote any such solution, even if not unique, as $\phi\left(t, x_{0}, d(\cdot)\right), t \in I(x, d(\cdot))$, where $I(x, d(\cdot))$ is its existence interval. Throughout the paper, $\|\cdot\|$ denotes the usual Euclidean norm, and "smooth" means $C^{\infty}$. For a differentiable function $V: \mathbb{R}^{n} \rightarrow \mathbb{R}$ the expression $L_{f_{d}} V(x)$ denotes the directional derivative $D V(x) f(x, d)$.

The general framework afforded by the model (1) allows us to treat simultaneously classical differential equations (the case when $D=\{0\}$ ) and more generally robust stability of differential equations subject to perturbations (when functions in $\mathcal{D}$ are seen as disturbances which do not change the equilibrium, as in parameter uncertainty), as well as systems with inputs in which elements of $\mathcal{D}$ are seen as exogenous tracking or regulation signals, or as actuator errors (in which case, the continuity properties of $(x, d) \mapsto \phi(\cdot, x, d)$ are of interest). In light of these applications, we now describe the appropriate stability concepts.

For the first, assume that $D$ is compact and that $f(0, d)=0$ for all $d \in D$. Then we say that the zero state is uniformly globally asymptotically stable (UGAS) if there exists a class $\mathcal{K} \mathcal{L}$ function $\beta$ such that, for each $d(\cdot) \in \mathcal{D}$, every maximal solution is defined for all $t \geq 0$ and

$$
\|\phi(t, x, d(\cdot))\| \leq \beta(\|x\|, t)
$$

for all $t \geq 0$. As usual, we call a function $\alpha:[0, \infty) \rightarrow[0, \infty)$ of class $\mathcal{K}$, if it satisfies $\alpha(0)=0$ and is continuous and strictly increasing (and class $\mathcal{K}_{\infty}$ if it is unbounded), and we call a continuous function $\beta:[0, \infty)^{2} \rightarrow[0, \infty)$ of class $\mathcal{K} \mathcal{L}$, if it is decreasing to zero in the second and of class $\mathcal{K}$ in the first argument. (It is an easy exercise, cf. e.g. [20], to verify that this definition is equivalent to the requirements of uniform stability and uniform attraction stated in " $\varepsilon-\delta$ " terms.) Note that while our general assumptions on the right hand side $f$ do not guarantee uniqueness of solutions through zero, the added assumption of asymptotic stability implies that $\phi(t, 0, d) \equiv 0$ is the unique solution with initial condition $x=0$, for all $d \in \mathcal{D}$. As a consequence, since away from zero we have a local Lipschitz condition, solutions are unique for each given initial state and $d \in \mathcal{D}$.

If the origin is no common fixed point for all values $d \in D$ then (2) is impossible. In this case, however, still a useful notion of stability is possible. We call the system (1) (globally) 
input-to-state stable (ISS), if there exists a class $\mathcal{K} \mathcal{L}$ function $\beta$ and a class $\mathcal{K}_{\infty}$ function $\alpha$ such that all solutions of (1) satisfy

$$
\|\phi(t, x, d(\cdot))\| \leq \beta(\|x\|, t)+\alpha\left(\sup _{0 \leq \tau \leq t}\|d(\tau)\|\right)
$$

for all $d(\cdot) \in \mathcal{D}$ and all $t \geq 0$. Formulation (3) is the most frequently used characterization of the ISS property. Note that with $\tilde{\beta}=2 \beta$ and $\tilde{\alpha}=2 \alpha$ inequality (3) immediately implies

$$
\|\phi(t, x, d(\cdot))\| \leq \max \left\{\tilde{\beta}(\|x\|, t), \tilde{\alpha}\left(\sup _{0 \leq \tau \leq t}\|d(\tau)\|\right)\right\}
$$

hence this "max" formulation can be used as an equivalent characterization.

Two apparently stronger formulations of these properties are obtained if we replace $\beta(\|x\|, t)$ by $c e^{-\lambda t}\|x\|$, more precisely we call the zero position of (1) uniformly globally exponentially stable (UGES), if there exist constants $c \geq 1, \lambda>0$ such that

$$
\|\phi(t, x, d(\cdot))\| \leq c e^{-\lambda t}\|x\|
$$

holds for all $d(\cdot) \in \mathcal{D}$ and all $t \geq 0$, and we call the system input-to-state exponentially stable (ISES), if there exist a class $\mathcal{K}_{\infty}$ function $\alpha$ and constants $c \geq 1, \lambda>0$ such that

$$
\|\phi(t, x, d(\cdot))\| \leq \max \left\{c e^{-\lambda t}\|x\|, \alpha\left(\sup _{0 \leq \tau \leq t}\|d(\tau)\|\right)\right\}
$$

for all $d(\cdot) \in \mathcal{D}$ and all $t \geq 0$. (As usual, these definitions use appropriate constants $c, \lambda>0$. In this paper, however, we will see that we can always work with "normalized" versions choosing $c=1, \lambda=1$. For the (ISES) property we use the "max" formulation because it allows a further implication as stated in Theorem 5, below. Observe that (5) implies (3) with $\left.\beta(\|x\|, t)=c e^{-\lambda t}\|x\|.\right)$

Extending the concepts in [1, p. 207] to our nonlinear setting, we will call a homeomorphism

$$
T: \mathbb{R}^{n} \rightarrow \mathbb{R}^{n}
$$

a change of variables if $T(0)=0, T$ is $C^{1}$ on $\mathbb{R}^{n}$, and $T$ is diffeomorphism on $\mathbb{R}^{n} \backslash\{0\}$ (i.e., the restrictions of $T$ and of $T^{-1}$ to $\mathbb{R}^{n} \backslash\{0\}$ are both smooth). Given a change of variables $T$ and a system (1), we may consider the transformed system

$$
\dot{y}(t)=\tilde{f}(y(t), d(t)),
$$

where, by definition,

$$
\tilde{f}(y, d)=D T\left(T^{-1}(y)\right) f\left(T^{-1}(y), d\right) .
$$


In other words, system (6) is obtained from the original system by means of the change of variables $y=T(x)$. Observe that the new system again satisfies the general requirements: $\tilde{f}(y, d)$ is continuous, and it is locally Lipschitz on $x$ for $x \neq 0$, uniformly on $d$.

It is our aim to show that for dimensions $n \neq 4,5$ the following assertions are true. Given a system of the form (1) satisfying (2) or (3), respectively, there exists a transformed system that satisfies (4) or (5), respectively. In this sense, global asymptotic stability is equivalent to global exponential stability under nonlinear changes of coordinates. Furthermore, one may obtain transformed systems where the constants defining the exponential stability property can be chosen to be the special values $c=\lambda=1$.

Furthermore we show that if system (1) is ISES (5) with $c=\lambda=1$ then there exists a homeomorphism $R: \mathbb{R}^{m} \rightarrow \mathbb{R}^{m}$ on the input space with $R(0)=0$ that is a diffeomorphism on $\mathbb{R}^{m} \backslash\{0\}$ such that the transformed system with $v=R(d)$

$$
\dot{y}(t)=\bar{f}(x(t), v(t)), \quad \bar{f}(x, v)=f\left(x, R^{-1}(v)\right)
$$

satisfies the following " $L_{2}$ to $L_{2}$ " nonlinear $H_{\infty}$ estimate:

$$
\int_{0}^{t}\|\phi(s, x, v(\cdot))\|^{2} d s \leq\|x\|^{2}+\int_{0}^{t}\|v(s)\|^{2} d s .
$$

Since (8) in turn implies ISS (by [31, Theorem 1]), we obtain equivalence between ISS and the nonlinear $H_{\infty}$ estimate (8) up to nonlinear changes of coordinates.

\section{Construction of the coordinate transformation}

The main tool for our construction of $T$ is the use of an appropriate Lyapunov function $V$. In fact, we can obtain $T$ for a whole class of functions as stated in the following proposition. Recall that a function $V: \mathbb{R}^{n} \rightarrow \mathbb{R}$ is called positive definite if $V(0)=0$ and $V(x)>0$ for all $x \neq 0$, and proper if the set $\{x \mid V(x) \leq \beta\}$ is bounded for each $\beta \geq 0$.

The next result says in particular that any such function may look like $\|x\|^{2}$ under a coordinate change. This implies in particular that the level sets under coordinate change are spheres. It may therefore not come as a surprise that a basic ingredient of the proof is related to the question of whether level sets of Lyapunov functions in $\mathbb{R}^{n}$ are diffeomorphic to the sphere $S^{n-1}$. This question is solved except for the two special cases of dimensions $n=4$ and $n=5$, though in the case $n=5$ it is at least known that the statement is true if only homeomorphisms are required. (For the case $n=4$ this question is equivalent to the Poincaré conjecture; see [36].)

Proposition 1 Let $n \neq 4$ and let $V: \mathbb{R}^{n} \rightarrow \mathbb{R}$ be a proper, positive definite $C^{1}$ function. Assume furthermore that $V$ is smooth on $\mathbb{R}^{n} \backslash\{0\}$ with nonvanishing gradient. Then for each 
class $\mathcal{K}_{\infty}$ function $\gamma$ which is smooth on $(0, \infty)$ there exists a homeomorphism $T: \mathbb{R}^{n} \rightarrow \mathbb{R}^{n}$ with $T(0)=0$ such that

$$
\tilde{V}(y):=V\left(T^{-1}(y)\right)=\gamma(\|y\|)
$$

In particular this holds for $\gamma(\|y\|)=\|y\|^{2}$.

If $n \neq 4,5$ then $T$ can be chosen to be a diffeomorphism on $\mathbb{R}^{n} \backslash\{0\}$. Furthermore, in this case there exists a class $\mathcal{K}_{\infty}$ function $\gamma$ which is smooth on $(0, \infty)$ and satisfies $\gamma(s) / \gamma^{\prime}(s) \geq s$ such that $T$ is $C^{1}$ with $D T(0)=0$.

PROOF. For the function $V$ the right hand side of the normed gradient flow

$$
\dot{x}=\frac{\nabla V(x)^{\prime}}{\|\nabla V(x)\|^{2}}
$$

is well defined and smooth for $x \neq 0$. Denote the solutions by $\psi(t, x)$. Then $V(\psi(t, x))=$ $V(x)+t$, and thus since $V$ is proper and $\nabla V(x) \neq 0$ for $x \neq 0$ for a given initial value $x \in \mathbb{R}^{n}$ $\psi$ is well defined for all $t \in(-V(x), \infty)$, thus also smooth (see e.g. [10, Corollary 4.1]).

Fix $c>0$. We define a map $\pi: \mathbb{R}^{n} \backslash\{0\} \rightarrow V^{-1}(c)$ by

$$
\pi(x)=\psi(c-V(x), x) .
$$

Obviously $\pi$ is smooth, and since the gradient flow crosses each level set $V^{-1}(a), a>0$ exactly once it induces a diffeomorphism between each two level sets of $V$, which are $C^{\infty}$ manifolds due to the fact that $V$ is smooth away from the origin with nonvanishing gradient.

Now observe that the properties of $V$ imply that $V^{-1}(c)$ is a homotopy sphere (cf. also [36, Discussion after Theorem 1.1]), which implies that $V^{-1}(c)$ is diffeomorphic to $\mathbb{S}^{n-1}$ for $n=1,2,3$ (see e.g. [22, Appendix] for $n=2$, [7, Theorem 3.20] for $n=3 ; n=1$ is trivial). For $n \geq 6$ we can use the fact that the sublevel set $\left\{x \in \mathbb{R}^{n} \mid V(x) \leq c\right\}$ is a compact, connected smooth manifold with a simply connected boundary, which by $[21, \S 9$, Proposition A] implies that the sublevel set is diffeomorphic to the unit disc $D^{n}$, hence $V^{-1}(c)$ is diffeomorphic to $\mathbb{S}^{n-1}$. Thus for all dimensions $n \neq 4,5$ we may choose a diffeomorphism $S: V^{-1}(c) \rightarrow \mathbb{S}^{n-1}$. By [8] we may choose $S$ to be at least a homeomorphism in the case $n=5$.

Let $Q:=S \circ \pi$. The coordinate transformation $T$ is now given by $T(0)=0$ and

$$
T(x)=\gamma^{-1}(V(x)) Q(x), \quad x \neq 0 .
$$

An easy computation verifies that $T^{-1}(0)=0$ and

$$
T^{-1}(y)=\psi\left(\gamma(y)-c, S^{-1}\left(\frac{y}{\|y\|}\right)\right), \quad y \neq 0
$$


hence $T$ is a diffeomorphism on $\mathbb{R}^{n} \backslash\{0\}$ (resp. a homeomorphism if $n=5$ ). Since $V(0)=0$, and $\psi\left(t, S^{-1}(y /\|y\|)\right) \rightarrow 0$ as $t \searrow-c$, both $T$ and $T^{-1}$ are homeomorphisms.

Finally, we have that

$$
\begin{aligned}
V\left(T^{-1}(y)\right) & =V\left(\psi\left(\gamma(\|y\|)-c, S^{-1}\left(\frac{y}{\|y\|}\right)\right)\right) \\
& =V\left(S^{-1}\left(\frac{y}{\|y\|}\right)\right)-c+\gamma(\|y\|)=\gamma(\|y\|)
\end{aligned}
$$

which finishes the proof of the first assertion.

For $n \neq 4,5$ and $s>0$ we define

$$
L(s):=\sup _{V(x)=s}\|D Q(x)\|
$$

and choose any class $\mathcal{K}$ function $a$ which is $C^{\infty}$ and satisfies

$$
a(s) \leq \frac{s}{L(s)} \text { for all } s \in(0,1]
$$

Then the function $h$ given by

$$
h(r)=\int_{0}^{r} a(s) d s
$$

is smooth and of class $\mathcal{K}_{\infty}$. Note that this construction implies $h(r) \leq r a(r)$ for all $r \geq 0$, hence $h(r) / h^{\prime}(r) \leq r$. Thus $\gamma:=h^{-1}$ is of class $\mathcal{K}_{\infty}$, smooth on $(0, \infty)$, and satisfies

$$
\frac{\gamma(s)}{\gamma^{\prime}(s)}=h^{-1}(s) h^{\prime}\left(h^{-1}(s)\right) \geq \frac{h^{-1}(s) h\left(h^{-1}(s)\right)}{h^{-1}(s)}=s .
$$

Differentiating $T$ yields

$$
D T(x)=h^{\prime}(V(x)) Q(x) \cdot D V(x)+h(V(x)) D Q(x) .
$$

For $x \rightarrow 0$ the first term tends to 0 since both $h^{\prime}(V(x))=a(V(x))$ and $D V(x)$ tend to 0 , and the second tends to 0 since for all $x$ sufficiently close to 0 the inequality

$$
h(V(x))\|D Q(x)\| \leq a(V(x))\|D Q(x)\| \leq \frac{V(x)}{L(V(x))}\|D Q(x)\| \leq V(x)
$$

holds by construction of $h$. Thus $D T(x) \rightarrow 0$, as $x \rightarrow 0$, and consequently $T \in C^{1}$ with $D T(0)=0$, by a straightforward application of the mean value theorem, see e.g. [19, Chap. 
$\mathrm{V}$, Theorem 3.2] and the fact that a function is continuously differentiable if all partial derivatives exist and are continuous.

\section{Main Results}

Using the coordinate transformation $T$ we can now prove our main results.

Theorem 2 Let $n \neq 4,5$ and consider any system (1) on $\mathbb{R}^{n}$ which is UGAS (2). We suppose that the set $D \subset \mathbb{R}^{m}$ is compact. Then, (1) can be transformed into a system (6) that is UGES (4).

In particular, the constants in (4) can be chosen to be $c=1, \lambda=1$.

PROOF. Under our assumptions, by [20, Theorem 2.9, Remark 4.1] ${ }^{*}$ there exists a smooth function $V: \mathbb{R}^{n} \rightarrow \mathbb{R}$ for (1) such that

$$
L_{f_{d}} V(x) \leq-\alpha_{1}(\|x\|)
$$

for some class $\mathcal{K}_{\infty}$ function $\alpha_{1}$. Furthermore, there exist class $\mathcal{K}_{\infty}$ functions $\alpha_{2}, \alpha_{3}$ such that

$$
\alpha_{2}(\|x\|) \leq V(x) \leq \alpha_{3}(\|x\|)
$$

Now let $\alpha_{4}$ be a $C^{1}$ function of class $\mathcal{K}_{\infty}$ which is smooth on $(0, \infty)$ and satisfies $\alpha_{4}^{\prime}(0)=0$, such that $\alpha_{4}(a) \leq \min \left\{a, \alpha_{1} \circ \alpha_{3}^{-1}(a)\right\}$ for all $a \geq 0$.

Such a function can be obtained e.g. by a slight modification of the construction in [26, Proof of Lemma 11]: Take a class $\mathcal{K}_{\infty}$ function satisfying $\delta(a) \leq \min \left\{a, \alpha_{1} \circ \alpha_{3}^{-1}(a)\right\}$ and which is smooth on $(0, \infty)$. Then

$$
\alpha_{4}(a)=\frac{2}{\pi} \int_{0}^{a} \frac{\delta(\tau)}{1+\tau^{2}} d \tau
$$

has the desired properties. Thus we obtain

$$
L_{f_{d}} V(x) \leq-\alpha_{4}(V(x))
$$

Now define

$$
\rho(a):=\exp \left(-\int_{a}^{1} \alpha_{4}(\tau)^{-1} d \tau\right) \text { for } a>0, \quad \rho(0):=0
$$

* To be precise, the results in that reference make as a blanket assumption the hypothesis that $f$ is locally Lipschitz, not merely continuous, at $x=0$. However, as noted in e.g. [35], the Lipschitz condition at the origin is not used in the proofs. 
Obviously $\rho$ is smooth on $(0, \infty)$; furthermore $\rho$ is of class $\mathcal{K}_{\infty}$ and by [26, Lemma 12] $\rho$ is a $C^{1}$ function on $[0, \infty)$ with $\rho^{\prime}(0)=0$. Thus defining

$$
W(x):=\rho(V(x))
$$

we obtain a $C^{1}$ Lyapunov function, which is smooth on $\mathbb{R}^{n} \backslash\{0\}$, for which an easy calculation shows that

$$
L_{f_{d}} W(x)=\frac{\exp \left(-\int_{V(x)}^{1} \alpha_{4}(\tau)^{-1} d \tau\right)}{\alpha_{4}(V(x))} L_{f_{d}} V(x) \leq-W(x)
$$

Applying Proposition 1 to $W$, using the class $\mathcal{K}_{\infty}$ function $\gamma$ with $\gamma(s) / \gamma^{\prime}(s) \geq s$ we obtain for each $d \in D$ and $y \neq 0$

$$
\langle\tilde{f}(y, d), y\rangle=\frac{\|y\|}{\gamma^{\prime}(\|y\|)} L_{\tilde{f}_{d}} \tilde{W}(y) \leq-\frac{\|y\|}{\gamma^{\prime}(\|y\|)} \tilde{W}(y)=-\frac{\|y\|}{\gamma^{\prime}(\|y\|)} \gamma(\|y\|) \leq-\|y\|^{2} .
$$

Clearly the overall inequality also holds for $y=0$ so that we obtain

$$
\frac{d}{d t}\|y(t)\|^{2}=2\langle\tilde{f}(y(t), d(t)), y(t)\rangle \leq-2\|y(t)\|^{2}
$$

and hence $\|y(t)\|^{2} \leq e^{-2 t}\|y(0)\|^{2}$, i.e. the desired exponential estimate.

Theorem 3 Let $n \neq 4,5$ and suppose that the system (1) on $\mathbb{R}^{n}$ is ISS (3) with some class $\mathcal{K}_{\infty}$ function $\alpha$ and some class $\mathcal{K} \mathcal{L}$ function $\beta$. Then (1) is can be transformed into a system (6) that is ISES (5) with constants $c=\lambda=1$.

PROOF. By $[32 \text {, Theorem } 1]^{\dagger}$ there exists a $C^{1}$ function $V$ which is smooth on $\mathbb{R}^{n} \backslash\{0\}$ and a class $\mathcal{K}_{\infty}$ function $\chi$ such that

$$
\|x\|>\chi(\|d\|) \quad \Rightarrow \quad L_{f_{d}} V(x) \leq-\alpha_{1}(\|x\|)
$$

for some class $\mathcal{K}_{\infty}$ function $\alpha_{1}$. Furthermore, there exist class $\mathcal{K}_{\infty}$ functions $\alpha_{2}, \alpha_{3}$ such that

$$
\alpha_{2}(\|x\|) \leq V(x) \leq \alpha_{3}(\|x\|)
$$

As in the proof of Theorem 2 we find a function $\rho$ which is class $\mathcal{K}_{\infty}, C^{1}$, and smooth on $\mathbb{R}^{n} \backslash\{0\}$, such that $W=\rho \circ V$ satisfies

$$
\|x\|>\chi(\|d\|) \quad \Rightarrow \quad L_{f_{d}} W(x) \leq-W(x) .
$$

$\dagger$ As with the UGAS proof, it is easy to verify that the assumption that the right-hand side is Lipschitz at zero is never actually used in [32]. The possible non-uniqueness of trajectories does not affect the argument used in Lemma 2.12 in that paper, which reduces the problem to one of UGAS. 
Now Proposition 1 yields a parameter transformation $T$ such that $\tilde{W}(y)=W\left(T^{-1}(y)\right)=$ $\gamma(\|y\|)$ and $\gamma(s) / \gamma^{\prime}(s) \geq s$.

Now choose a class $\mathcal{K}_{\infty}$ function $\delta$ such that $\left\|T^{-1}(y)\right\| \geq \delta(\|y\|)$ and define $\tilde{\alpha}=\delta^{-1} \circ \chi$. Then a straightforward calculation yields

$$
\|y\|>\tilde{\alpha}(\|d\|) \quad \Rightarrow \quad L_{\tilde{f}_{d}} \tilde{W}(y) \leq-\tilde{W}(y) .
$$

Similar to the proof of Theorem 2 this implies

$$
\|\tilde{\phi}(t, y, d(\cdot))\| \leq e^{-t}\|y\|
$$

as long as $\|\tilde{\phi}(t, y, d(\cdot))\|>\tilde{\alpha}\left(\sup _{0 \leq \tau \leq t}\|d(\tau)\|\right)$ which yields the desired estimate.

Theorem 4 Consider the system (1) on $\mathbb{R}^{n}$ being ISES (5) with some class $\mathcal{K}_{\infty}$ function $\alpha$ and $c=\lambda=1$. Then there exists a homeomorphism $R: \mathbb{R}^{m} \rightarrow \mathbb{R}^{m}$ on the input space with $R(0)=0$, that is a diffeomorphism on $\mathbb{R}^{m} \backslash\{0\}$, such that the the transformed system (7) satisfies the nonlinear $H_{\infty}$ estimate (8).

PROOF. From (5) it is immediate that for any $d(\cdot) \in \mathcal{D}$, any $x \in \mathbb{R}^{n}$, and any $T>0$ we have

$$
\|x\| \geq e^{T} \alpha\left(\sup _{0 \leq \tau \leq T}\|d(\tau)\|\right) \quad \Rightarrow \quad\|\phi(t, x, d(\cdot))\| \leq e^{-t}\|x\| \text { for all } t \in[0, T]
$$

Now consider the function $W(x)=\|x\|^{2}$. Then (13) implies

$$
\|x\| \geq e^{T} \alpha\left(\sup _{0 \leq \tau \leq T}\|d(\tau)\|\right) \quad \Rightarrow \quad W(\phi(t, x, d(\cdot))) \leq e^{-2 t} W(x) \text { for all } t \in[0, T] .
$$

In particular this estimate is valid for constant functions $d(\cdot) \equiv d \in D$, thus the mean value theorem (observe $\left.W(\phi(0, x, d))=W(x)=e^{-0} W(x)\right)$ yields

$$
\|x\| \geq \alpha(\|d\|) \quad \Rightarrow \quad L_{f_{d}} W(x) \leq-2 W(x) \leq-W(x) .
$$

Now defining

$$
\tilde{\alpha}(r)=\sup _{\|x\| \leq \alpha(r),\|d\| \leq r}\langle f(x, d), x\rangle
$$

we obtain a class $\mathcal{K}_{\infty}$ function $\tilde{\alpha}$ with

$$
L_{f_{d}} W(x) \leq-W(x)+\tilde{\alpha}(\|d\|) .
$$


Without loss of generality (one could take a larger $\tilde{\alpha}$ ), we may assume $\tilde{\alpha}$ to be smooth on $(0, \infty)$, and thus

$$
R(d):=\frac{\tilde{\alpha}(\|d\|)^{2} d}{\|d\|}
$$

has the regularity properties as stated in the assertion. Now the transformation (7) yields

$$
L_{\bar{f}_{v}} W(x) \leq-W(x)+\|v\|^{2} .
$$

Integrating this equation along a trajectory $x(\cdot)$ gives

$$
W(x(t))-W(x(0)) \leq-\int_{0}^{t} W(x(s)) d s+\int_{0}^{t}\|v(s)\|^{2} d s
$$

which implies (8) since $W(x)=\|x\|^{2}$.

\section{Remarks}

Note that, in general, for our results to be true we cannot expect $T$ to be diffeomorphic on the whole $\mathbb{R}^{n}$. Consider the simplest case where $f$ does not depend on $d$ and is differentiable at the origin. If $T$ were a diffeomorphism globally, then $D T^{-1}(0)$ would be well-defined, which implies that

$$
D \tilde{f}(0)=\left.\frac{\partial}{\partial y}\right|_{y=0} D T\left(T^{-1}(y)\right) f\left(T^{-1}(y)\right)=D T(0) D f(0) D T^{-1}(0)
$$

and so the linearizations in 0 are similar; in particular, the dimension of center manifolds remains unchanged.

Actually, if one wants the exponential decay to be $e^{-t}$, even for linear systems one cannot obtain a diffeomorphism $T$. As an example, consider the one-dimensional system $\dot{x}=-x / 2$. Here one uses the change of variables $y=T(x)$ given by $T(x)=x^{2}, x>0, T(0)=0$ and $T(x)=-x^{2}, x<0$ to obtain $\dot{y}=-y$. Note that $T$ is $C^{1}$ with $D T(0)=0$. The inverse of this $T$ is given by $T^{-1}(y)=\sqrt{y}, y>0, T^{-1}(0)=0$ and $T^{-1}(y)=-\sqrt{-y}, y<0$ which is smooth only away from the origin, though continuous globally.

An example for the case of nontrivial center manifolds is given by the system $\dot{x}=-x^{3}$. Let us first note that for this system there is no transformation in the class we consider such that the transformed system is of the form $\dot{y}=-y$. The reason for this is that we would have $\dot{T}(x)=$ $\dot{y}=-y=-T(x)$, so at least for $x>0 \mathrm{~V}=T$ is a Lyapunov function with the property that $\dot{V}(x)=-V^{\prime}(x) x^{3}=-V(x)$. It is readily seen that the solutions of this differential equation (in $x$ and $V$ ) are $V_{c}(x)=c \exp \frac{1}{-2 x^{2}}$, for $c \in \mathbb{R}$. However, the image of $[0, \infty)$ under such 
$V_{c}$ yields a bounded set, so that these functions are no candidate for coordinate transforms on $\mathbb{R}$. Nonetheless a coordinate transform according to our requirements can now be easily built: Take any $\mathcal{K}_{\infty}$ function $\alpha$ with $\alpha^{\prime}>0$ on $(0, \infty)$ so that with via the symmetrization $\alpha(-x):=\alpha(x)$ we get a smooth function on $\mathbb{R}$. Now define

$$
T(x):=\alpha(x) V_{1}(x), x \geq 0, \quad T(x):=-\alpha(x) V_{1}(x), x<0 .
$$

Then for $y \neq 0$ we have $\dot{y}=T \dot{(x)}=-\left(1+\frac{\alpha^{\prime}(x) x^{3}}{\alpha(x)}\right) T(x)<-y$, so that the transformed system decays at least exponentially with constants $c=1, \lambda=1$. Again note that the requirement $D T(0)=0$ is vital, in fact all orders of derivatives vanish in 0 .

A basic ingredient of the proof of Theorem 2 is the construction of a Lyapunov function with the property $\dot{V} \leq-V$. Actually, one may even, under restricted conditions, obtain the equality $\dot{V}=-V$. It should be noted that already in [3] it is shown that for dynamical systems with globally asymptotically stable fixed point a continuous Lyapunov function with the property $V(\phi(t, x))=e^{-t} V(x)$ exists, see also Chapter V.2 in [4]. Note, however that in these references only systems with trajectories defined on $\mathbb{R}$ are considered, which does not include the previous example. Indeed, if $f(x, d)=f(x)$ is independent of $d \in D$ and the system $\dot{x}=f(x)$ is backward complete we can can also define a coordinate transformation based on a different $W$ than the one used in the proof of Theorem 2: In this case the function $W(x)=\exp t(x)$ with $t(x)$ defined by $V(\phi(t(x), x))=1$ is positive definite, proper, and satisfies $L_{f} W(x)=-W(x)$, thus $W(\phi(t, x))=W(x)-t$. Since $V^{-1}(1)=W^{-1}(1)$ we still find a diffeomorphism $S$ as in the proof of Proposition 1. Deviating from this proof, instead of the gradient flow we now use the trajectories of the system, i.e. we define $\pi(x)=\phi(W(x)-1, x)$ yielding $W(\pi(x))=W(x)-(W(x)-1)=1$. Thus from $\pi$ we can construct $T$ as in the proof of Proposition 1, and obtain $W\left(T^{-1}(y)\right)=\|y\|^{2}$. Furthermore the definition of $\pi$ implies that each trajectory $\{\phi(t, x) \mid t \in \mathbb{R}\}$ is mapped onto the line $\{\alpha S(\pi(x)) \mid \alpha>0\}$ and consequently $\tilde{f}(y)=-y$, i.e. we obtain a transformation into the linear system $\dot{y}=-y$. Note, however, that with this construction the coordinate transformation will in general only have the regularity of $f$ (e.g. a homeomorphism if $f$ is only $C^{0}$ ), which is inevitable since it transforms $f$ into a smooth map. Moreover, this construction cannot be generalized to systems with disturbances.

Since we are not requiring that the inverse of a change of variables be itself a change of variables (because one may, and in fact does in our constructions, have $D T(0)=0$, in which case $T^{-1}$ is not differentiable at the origin), the way to define a notion of "equivalence" is by taking the transitive and symmetric closure of the relation given by such changes of variables. That is, we could say that system (1) is equivalent to a system (6) if there exist $k \in \mathbb{N}$ and maps $f_{0}=f, f_{1}, \ldots, f_{k}=\tilde{f}: \mathbb{R}^{n} \times D \rightarrow \mathbb{R}^{n}$, all satisfying the assumptions on $f$, with the following properties: For each $i=0, \ldots, k-1$ there exists a change of variables $T$ as above such that $f_{l}(y, d)=D T\left(T^{-1}(y)\right) f_{m}\left(T^{-1}(y), d\right)$, where $l=i, m=i+1$ or $l=i+1, m=i$.

Finally, regarding our notion of system transformation, note that even if $f(0, d) \neq 0$ for some $d \in \mathcal{D}$ for the original system (1), then under the assumption $D T(0)=0$ we have $\tilde{f}(0, d)=0$ for all $d \in \mathcal{D}$ for the transformed system. This implies that even if the original system had unique trajectories through zero, the transformed system cannot have this property. 
Acknowledgments: We thank David Angeli for suggestions regarding the remark on backward complete systems, as well as Uwe Helmke and Laurent Praly for many references to the literature.

\section{References}

[1] V.I. Arnol'd. Ordinary Differential Equations, Springer-Verlag, Berlin, 1992.

[2] T. Başar and P. Bernhard. $H^{\infty}$-Optimal Control and Related Minimax Design Problems. A Dynamic Game Approach, Second edition, Birkhäuser, Boston, 1995.

[3] N. Bhatia. On asymptotic stability in dynamical systems. Math. Syst. Theory 1(1967): 113-127.

[4] N. Bhatia and G. Szegö. Stability Theory of Dynamical Systems. Number 161 in Grundlehren der mathematischen Wissenschaften. Springer-Verlag, Berlin, New York, Heidelberg, 1970.

[5] P.D. Christofides and A.R. Teel. Singular perturbations and input-to-state stability. IEEE Trans. Automat. Control 41(1996): 1645-1650.

[6] C. Coleman. Local trajectory equivalence of differential systems. Proc. Amer. Math. Soc. 16(1965): 890-892. Addendum. Ibid. 17, 770 (1966).

[7] B.A. Dubrovin, A.T. Fomenko, and S.P. Novikov. Modern Geometry - Methods and Applications, Part III: Introduction to Homology Theory, Springer-Verlag, New York, 1990.

[8] M.H. Freedman. The topology of four-dimensional manifolds. J. Differ. Geom. 17(1982): 357453.

[9] L. Grüne. Input-to-state stability of exponentially stabilized semilinear control systems with inhomogeneous perturbations, System $\&$ Control Letters, to appear.

[10] P. Hartman. Ordinary Differential Equations, Birkhäuser, Basel, Second edition, 1982.

[11] J.W. Helton and M.R. James. Extending $H_{\infty}$ Control to Nonlinear Systems, SIAM, Philadelphia, 1998.

[12] A. Isidori and A. Astolfi. Disturbance attenuation and $H_{\infty}$-control via measurement feedback in nonlinear systems. IEEE Trans. Automat. Control 37(1992): 1283-1293.

[13] A. Isidori. Global almost disturbance decoupling with stability for non minimum-phase singleinput single-output nonlinear systems. Systems $\&$ Control Letters 28 (1996), pp. 115-122.

[14] Z.P. Jiang, A. Teel, and L. Praly. Small-gain theorem for ISS systems and applications. Mathematics of Control, Signals, and Systems, 7 (1994), pp. 95-120.

[15] M. Kawski. Geometric homogeneity and applications to stabilization. Nonlinear Control System Design Symposium (NOLCOS), Lake Tahoe, June 1995.

[16] H.K. Khalil. Nonlinear Systems, Prentice-Hall, Upper Saddle River, NJ, second ed., 1996.

[17] M. Krstić and H. Deng. Stabilization of Uncertain Nonlinear Systems, Springer-Verlag, London, 1998.

[18] M. Krstić, I. Kanellakopoulos, and P.V. Kokotović. Nonlinear and Adaptive Control Design, John Wiley \& Sons, New York, 1995. 
[19] S. Lang. A first course in calculus, fifth ed., Springer-Verlag, New York, 1986.

[20] Y. Lin, E.D. Sontag, and Y. Wang. A smooth converse Lyapunov theorem for robust stability. SIAM J. Control Optim. 34(1996): 124-160.

[21] J. Milnor. Lectures on the h-Cobordism Theorem, Princeton Mathematical Notes, Princeton Univ. Press, Princeton, NJ. 1965.

[22] J. Milnor. Topology from the Differentiable Viewpoint, University Press of Virginia, Charlottesville 1965.

[23] L. Perko. Differential Equations and Dynamical Systems, Second edition, Springer-Verlag, New York, 1996.

[24] L. Praly. Generalized weighted homogeneity and state dependent time scale for linear controllable systems. Proc. Conf. Decision and Control CDC97, San Diego, CA, 1998, 43424347.

[25] J.-B. Pomet and L. Praly. A result on robust boundedness. Systems \& Control Letters 10(1988): 83-92.

[26] L. Praly and Y. Wang. Stabilization in spite of matched unmodelled dynamics and an equivalent definition of input-to-state stability. Math. of Control, Signals, and Systems 9(1996): 1-33.

[27] L. Rosier. Etude de quelques problèmes de stabilisation. Thèse de Doctorat. E.N.S. de Cachan, Univ. Paris XI, 1993.

[28] R. Sepulchre, M. Jankovic, and P.V. Kokotović. Constructive Nonlinear Control. SpringerVerlag, Berlin, 1997.

[29] E.D. Sontag. Smooth stabilization implies coprime factorization. IEEE Trans. Automat. Contr. 34(1989): 435-443.

[30] E.D. Sontag. Mathematical Control Theory, Deterministic Finite Dimensional Systems, Second Edition, Springer-Verlag, New York, 1998.

[31] E.D. Sontag. Comments on integral variants of ISS. Systems $\&$ Control Letters 34(1998): 93100.

[32] E.D. Sontag and Y. Wang. On characterizations of the input-to-state stability property. Systems E Control Letters 24 (1995): 351-359.

[33] J. Tsinias. Input to state stability properties of nonlinear systems and applications to bounded feedback stabilization using saturation. ESAIM Control Optim. Calc. Var. 2(1997): 57-85.

[34] A. van der Schaft. $L_{2}$-Gain and Passivity Techniques in Nonlinear Control (Lecture Notes in Control and Information Sciences, 218), Springer-Verlag, London, 1996.

[35] Y. Wang, A converse Lyapunov theorem with applications to ISS-disturbance attenuation. In Proc. 13th IFAC World Congress, 1996, vol. E, pp. 79-84.

[36] F.W. Wilson. The structure of the level surfaces of a Lyapunov function. J. Differ. Equations 3 (1967): 323-329. 